\title{
Inhibitory Influence of the Dorsal Nucleus of the Lateral Lemniscus on Binaural Responses in the Rat's Inferior Colliculus
}

\author{
Liang Li and Jack B. Kelly \\ Laboratory of Sensory Neuroscience, Department of Psychology, Carleton University, Ottawa, Ontario K1S 5B6, Canada
}

The contribution of the dorsal nucleus of the lateral lemniscus (DNLL) to binaural processing was examined by recording single-unit activity in the rat's inferior colliculus before, during, and after a reversible block of the excitatory activity in DNLL by local injection of kynurenic acid. Recordings were made from the central nucleus of the inferior colliculus with glass micropipettes filled with 3 м sodium acetate. Kynurenic acid (2 $\mathrm{mm}$ in Locke's solution) was injected into the DNLL through one side of a double-barreled glass pipette. The other side of the pipette was filled with Locke's solution for making control injections and recording neural activity. Pressure injection of $0.7-2.0 \mu \mathrm{l}$ of kynurenic acid resulted in the complete cessation of tone-evoked responses in DNLL for periods of $45 \mathrm{~min}$ to $1 \mathrm{hr}$. Tone bursts were delivered separately to the two ears through headphones fitted to the external auditory meatus. Binaural responses in the inferior colliculus were determined by comparing the effects of monaural and binaural stimulation. Attention was focused on neurons that were excited by contralateral stimulation and inhibited by ipsilateral stimulation. Interaural intensity difference (IID) functions were generated by holding contralateral sound pressure constant at $10 \mathrm{~dB}$ above threshold while increasing ipsilateral sound pressure level. Prior to kynurenic acid injection, ipsilateral stimulation caused a pronounced suppression of contralaterally evoked single-unit activity. Unilateral injection of kynurenic acid into DNLL reduced the strength of binaural suppression in the contralateral inferior colliculus. In every cell tested, the IID curve in inferior colliculus was shifted following contralateral DNLL injection. The IID curve returned to normal after recovery of neural activity in DNLL. In contrast, no effect was seen in the inferior colliculus ipsilateral to the kynurenic acid injection. The Injection of Locke's solution into DNLL had no effect on IID curves in the inferior colliculus. These data suggest that the DNLL plays a role in binaural processing through an inhibitory influence on responses in the contralateral inferior colliculus.

Several lines of evidence suggest that the dorsal nucleus of the lateral lemniscus (DNLL) plays a role in binaural processing. First, most neurons in the DNLL are sensitive to binaural stimulation. Both binaural summation and suppression responses

Received Apr., 2, 1992; revised June 10, 1992; accepted June 17, 1992

This research was supported by the Natural Sciences and Engineering Research Council of Canada and by the Ontario Deafness Research Foundation. We thank Joan Mallet for technical assistance in preparation of photomicrographs.

Correspondence should be addressed to Jack B. Kelly, Laboratory of Sensory Neuroscience, 329 Life Science Building, Department of Psychology, Carleton University, Ottawa, Canada K1S 5B6.

Copyright (C) 1992 Society for Neuroscience $0270-6474 / 92 / 124530-10 \$ 05.00 / 0$ have been reported for the DNLL in the cat (Aitkin et al., 1970; Brugge et al., 1970). Second, neuroanatomical studies have shown that the DNLL is a major component of the acoustic chiasm, providing decussating fibers through the commissure of Probst that could be important for binaural interaction. The DNLL has projections to the contralateral DNLL as well as both ipsilateral and contralateral inferior colliculus (Goldberg and Moore, 1967; Beyerl, 1978; Adams, 1979; Brunso-Bechtold et al., 1981; Glendenning et al., 1981; Kudo, 1981; Zook and Casseday, 1982, 1987; Coleman and Clerici, 1987; Shneiderman et al., 1988; Hutson et al., 1991). The vast majority of neurons in the DNLL are GABAergic and are thought to serve an inhibitory function in establishing the laterality of responses in the auditory system (Adams and Mugnaini, 1984; Thompson et al., 1985; Moore and Moore, 1987; Roberts and Ribak, 1987; Glendenning and Baker, 1988). The projections from DNLL to the contralateral DNLL and inferior colliculus have axon terminals that contain pleomorphic synaptic vesicles usually associated with inhibitory function (Oliver and Shneiderman, 1989; Shneiderman and Oliver, 1989). The convergence of these inhibitory terminals and excitatory projections from other sources may provide an opportunity for binaural interaction in the inferior colliculus (Shneiderman et al., 1988). Third, physiological recordings from the central nucleus of the inferior colliculus have revealed local binaural interactions that may be mediated in part by DNLL. Intracellular recordings from neurons in the cat's inferior colliculus have shown that stimulation of one ear can produce an EPSP and stimulation of the other ear an IPSP in the same neuron (Nelson and Erulkar, 1966). These data demonstrate the presence of binaural interactions within the inferior colliculus itself. Furthermore, binaural responses in the inferior colliculus can be altered by local application of GABA blockers. Iontophoretic injection of the $\mathrm{GABA}_{\mathrm{A}}$ antagonist bicuculline reduces the extent of binaural suppression of excitatory-inhibitory (EI) responses in inferior colliculus in both rat and mustache bat (Faingold et al., 1989, 1991; Park and Pollak, 1992). It is likely that the binaural inhibition blocked by bicuculline in these studies originates at least in part from GABAergic neurons in the contralateral DNLL. Finally, kainic acid lesions of the DNLL alter the binaural response characteristics of evoked potentials recorded from contralateral auditory cortex in the rat (Glenn and Kelly, 1991, 1992). In the normal rat, the amplitude of the auditory cortical evoked response varies as a function of binaural time differences between paired clicks. The largest evoked response amplitudes are found in the hemisphere contralateral to the leading click. As the interaural time difference (ITD) is shifted in favor of the ipsilateral ear, the response is steadily reduced until it reaches around $50 \%$ of maximum amplitude. In animals with unilateral DNLL lesions, the reduction in response amplitude associated with binaural timing 
is greatly diminished in the hemisphere contralateral to the lesion. This effect has been attributed to the disruption of inhibitory projections from the DNLL to the contralateral DNLL and/or inferior colliculus.

The purpose of the present study was to investigate directly the role of DNLL in shaping binaural responses in the central nucleus of the inferior colliculus. Recordings were made from single neurons in the infcrior colliculus beforc, during, and after a reversible pharmacological block of neural activity in the DNLL by local injection of the excitatory amino acid antagonist kynurenic acid. The effects of blocking DNLL activity ipsilateral and contralateral to the recording site were examined, sometimes on the same inferior colliculus neuron. Binaural responses were characterized by interaural intensity difference (IID) functions obtained by manipulation of the relative balance of the sound pressure level of tones delivered to the two ears.

\section{Materials and Methods}

Physiological procedures. Recordings were made from 19 male Wistar albino rats (250-350 gm) obtained from Charles River Inc., St. Constant, Quebec. Animals were anesthetized with pentobarbital sodium $(60 \mathrm{mg} /$ $\mathrm{kg}$, i.p.) for initial surgical procedures. A state of areflexia was maintained throughout the experiment by supplemental injections of Equithesin $(0.5 \mathrm{ml} / \mathrm{kg}$, i.p.) given at intervals of approximately $45 \mathrm{~min}$ (Equithesin is a combination of $42.6 \%$ chloral hydrate and $9.6 \%$ pentobarbital sodium in an aqueous solution; see Sally and Kelly, 1992, for method of preparation).The animals were placed in a head holder that left the external meatus free so that damage to the tympanic membrane could be avoided. A midline incision was made in the scalp, the skin was retracted laterally, and two small craniotomies were made just anterior to lambda to permit insertion of recording electrodes into the inferior colliculus. Additional craniotomies were made more laterally to allow placement of the injection pipettes in the left and right lateral lemniscus. The animals were then placed in a soundproof room, and acoustic couplers, connected to sealed headphones, were fitted snugly into the left and right ear canals. Recording electrodes and injection pipettes were then lowered stereotaxically into the inferior colliculus and lateral lemniscus, respectively. Stereotaxic coordinates for inferior colliculus placements were $1.7-2.0 \mathrm{~mm}$ lateral, $0.4-0.5 \mathrm{~mm}$ rostral, and $3.0-5.0$ $\mathrm{mm}$ ventral to lambda with the electrodes in a vertical orientation. For lateral lemniscus placement, the pipettes were tilted $30^{\circ}$ relative to the sagittal plane. The coordinates were referenced to a point $6.7 \mathrm{~mm}$ lateral and $0.4-0.5 \mathrm{~mm}$ rostral to lambda. The injection pipette was lowered to a depth of $7.8 \mathrm{~mm}$ from the reference point.

The injection pipettes were constructed from double-barreled glass tubing pulled to a tip diameter of $20 \mu \mathrm{m}$ per barrel. One barrel, filled with Locke's solution, was used for recording multiple-unit evoked activity from the DNLL to refine electrode placements and monitor the effect of kynurenic acid injections. This barrel was also used for control injections of Locke's solution. At the end of an experiment, a solution of pontamine sky blue ${ }^{1}$ was added to the pipette while it was still in place and was injected into the brain as a marker for histological verification of electrode placements. The injection of this dye, which carries a negative electrical charge, was made iontophoretically by application of 8-10 $\mu \mathrm{A}$ negative current to the pipette over a period of $15-20 \mathrm{~min}$. The second barrel of the pipettc was filled with $2 \mathrm{~mm}$ kynurcnic acid (Sigma) in Locke's solution. Kynurenic acid, an excitatory amino acid antagonist, was pressure injected into the lateral lemniscus through a length of polyethylene tubing connecting the pipette with a $5 \mathrm{ml}$ syringe and a three-way stopcock. The amount of injection was controlled by monitoring the movement of the surface of the solution along a calibrated length of the injection pipette. An injection of 0.7-2.0 $\mu \mathrm{l} \mathrm{kynu-}$ renic acid was usually sufficient to cause the complete cessation of neural activity in DNLL as monitored at the pipette tip.

The recording electrodes were single-barreled glass micropipettes pulled to a tip diameter of $2 \mu \mathrm{m}$ and backfilled with $3 \mathrm{M}$ sodium acetate (1.9$2.3 \mathrm{M} \Omega$ ). The sodium acetate solution contained $2-4 \%$ pontamine sky blue, which was ejected iontophoretically to mark the position of the neuron from which recordings were made. Each experiment was based

'Pontamine sky blue can be purchased from K\&K Laboratories, Division of ICN Biomedical Inc., Cleveland, $\mathrm{OH} 44128$. on a series of recordings from a single, well-isolated neuron in the inferior colliculus. The electrical activity was amplified $10,000 \times$ by a BAK amplifier (model MDA-4) and monitored audiovisually by an oscilloscope and a loudspeaker system. Single units werc sclected for quantitative analysis by a BAK time window discriminator. Action potential waveforms were displayed on a storage oscilloscope to ensure that recordings were from single units, and when adequate isolation had been achieved, acceptance pulses were fed to a Tracor Northern signal averager (TN-1550) for spike count and display of poststimulus time histograms (PSTHs).

Acoustical stimulation. Pure tone pulses were produced by sine waves from a Hewlett Packard function generator (HP $3310 \mathrm{~A}$ ), shaped by two parallel Coulbourn Instruments electronic gates (S 84-04), and led through paired decade attenuators to the two channels of a stereo power amplifier. The shaped signals were $110 \mathrm{msec}$ in duration with $10 \mathrm{msec}$ rise and decay times. The tone pulse repetition rate was $1 / \mathrm{sec}$. The signals were transduced by Pioneer SE-50D stereo headphone drivers mounted in a customizcd housing. The scaled headphone housing opened into a small acoustic coupler whose speculum was inserted into the rat's external ear canal. The couplers were constructed so that a probe tube could be inserted along the length of the speculum with its tip centered at the opening within a few millimeters of the tympanic membrane. Probe tube measurements of the acoustic cross talk in the system, with the couplers and headphones in place, indicated $65 \mathrm{~dB}$ attenuation at frequencies up to $1.5 \mathrm{kHz}$ and at least $70 \mathrm{~dB}$ attenuation for all higher frequencies.

Neurons were isolated for study by advancing electrodes through the inferior colliculus while simultaneously presenting either monaural or binaural tone pulses. Attention was focused in this study exclusively on binaural responses in which the contralateral stimulus excited and ipsilateral stimulus inhibited action potentials, that is, binaural suppression (EI) responses. When such a response was found, the characteristic frequency (CF; frequency at which the neuron was most sensitive) and contralateral threshold at CF were determined. An interaural intensity difference (IID) curve was then generated by setting the contralateral tone level at $10 \mathrm{~dB}$ above threshold and manipulating the ipsilateral tone level in $10 \mathrm{~dB}$ steps to determine firing rate at various IID values. An attempt was made to determine IID functions before injection of kynurenic acid, immediately after injection, and after a period of recovery following the injection. If the duration of the recording permitted, separate IID functions were obtained following ipsilateral and contralateral injections. Also, in some cases, IID curves were obtained for control purposes after injection of Locke's solution without kynurenic acid. Usually, because the length of time required to complete a full series of manipulations was several hours, and because we wished to avoid complications associated with multiple drug injections, a separate animal was used for every neuron isolated and studied in this manner.

Histology. Following a recording session, pontamine sky blue was injected into the inferior colliculus and DNLL to mark the position of the electrode tips. The animal was then perfused through the heart with physiological saline and formalin. The brains were removed and sectioned in the frontal plane for histological analysis. The location of pontamine sky blue was established in unstained $40 \mu \mathrm{m}$ frozen sections. Alternate sections were sometimes stained with cresyl violet, but the staining procedure itself tended to wash out and obscure identification of the dye. For the purpose of illustration, the position of the electrode site was transposed onto a representative diagram taken from a scries of standard frontal sections through the rat's auditory midbrain.

\section{Results}

Location of electrode sites. Experiments were successfully completed on 20 neurons in the rat's inferior colliculus. The effects of contralateral DNLL injections were determined for 10 neurons, the effect of an ipsilateral injection was determined for 1 neuron, and the effects of both ipsilateral and contralateral injections were determined for 9 neurons. Data were selected for presentation from 13 representative neurons, 7 located in the left and 6 in the right inferior colliculus. Electrode positions in inferior colliculus and DNLL were marked by pontamine sky blue injections as illustrated in Figure 1. The location of the electrode tip in most cases was easily identified in unstained frontal sections through the auditory midbrain. 

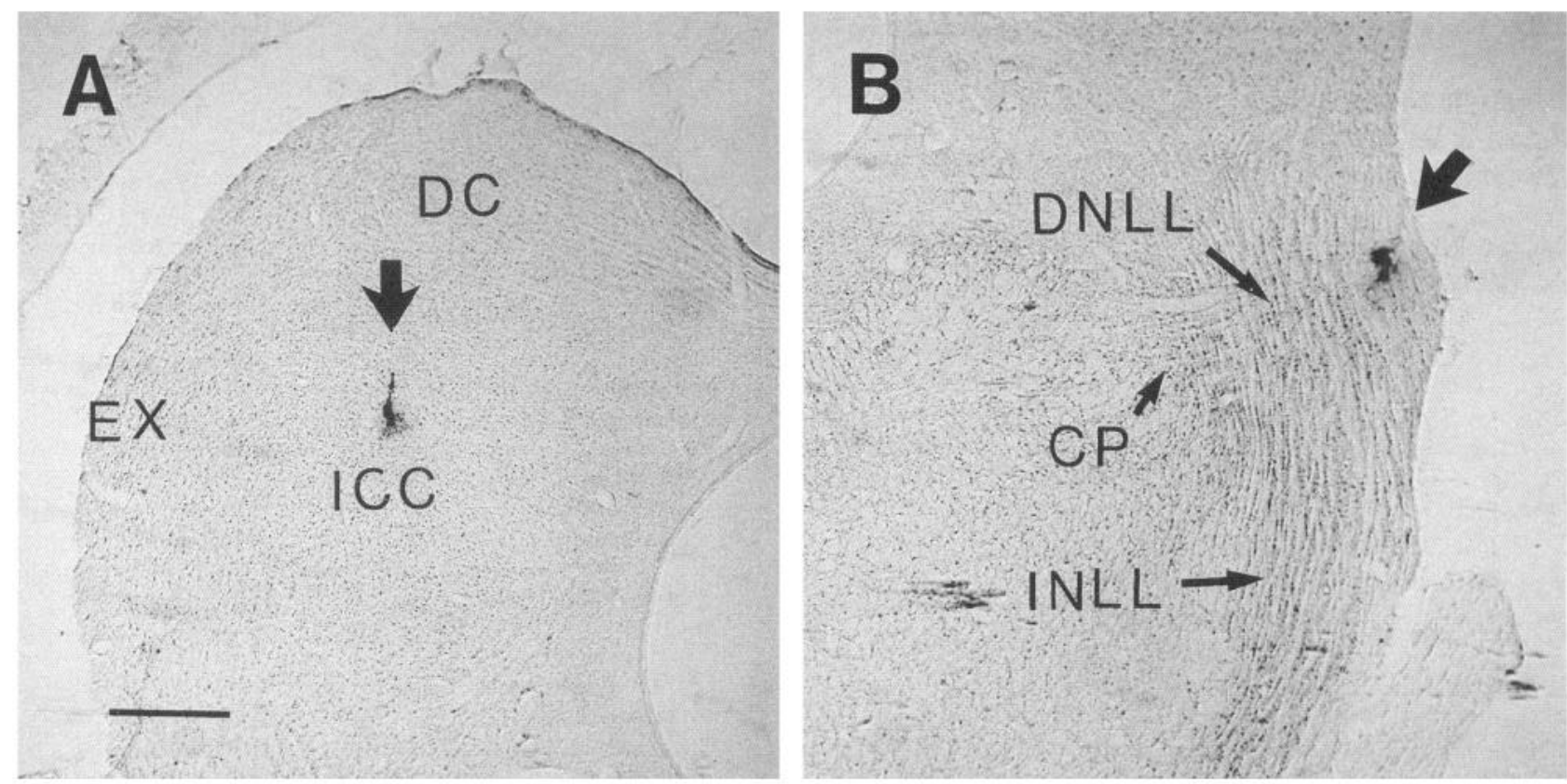

Figure 1. Examples of pontamine sky blue injections in the inferior colliculus $(A)$ and the DNLL $(B)$. The case illustrated here is cell $\mathrm{K}$ as shown in Figure 2. Photomicrographs were taken from unstained $40 \mu \mathrm{m}$ frontal sections viewed through a Zeiss $2.5 \times$ objective with the microscope condenser constricted to produce contrast enhancement. Pontamine sky blue injections are indicated by the large arrows. $C P$, commissure of Probst; $D C$, dorsal cortex of the inferior colliculus; $D N L L$, dorsal nucleus of the lateral lemniscus; $E X$, external nucleus of the inferior colliculus; $I C C$, central nucleus of inferior colliculus; $I N L L$, intermediate nucleus of the lateral lemniscus. Scale bar, $500 \mu \mathrm{m}$.

Because the dye was released directly from the tip of the recording electrode, the injection sites in inferior colliculus provide a clear indication of the location of cells from which recordings were made. The dye injections in DNLL, on the other hand, are approximations of the location of neurons influenced by kynurenic acid. The tip diameter of the injection pipette in DNLL was larger than the tip of the recording electrode in inferior colliculus ( $20 \mu \mathrm{m}$ compared to $2 \mu \mathrm{m})$. Also, the volume of kynurenic acid released was between 0.7 and $2.0 \mu$, which would result in considerable spread of drug away from the pipette tip. The deposit of dye shown in Figure $1 B$ was along the lateral margin of DNLL. Other dye injections penetrated more deeply into the DNLL as indicated in Figure 2. Although there is evidence that lateral and medial locations within DNLL have different projection patterns (Shneiderman et al., 1988), there was no indication in our study of effects related to pipette position, probably because of spread of kynurenic acid within DNLL.

A summary of the location of all electrode positions for which physiological data are presented is shown in Figure 2. The recording sites in inferior colliculus and the corresponding kynurenic acid injection sites in DNLL are identified by capital
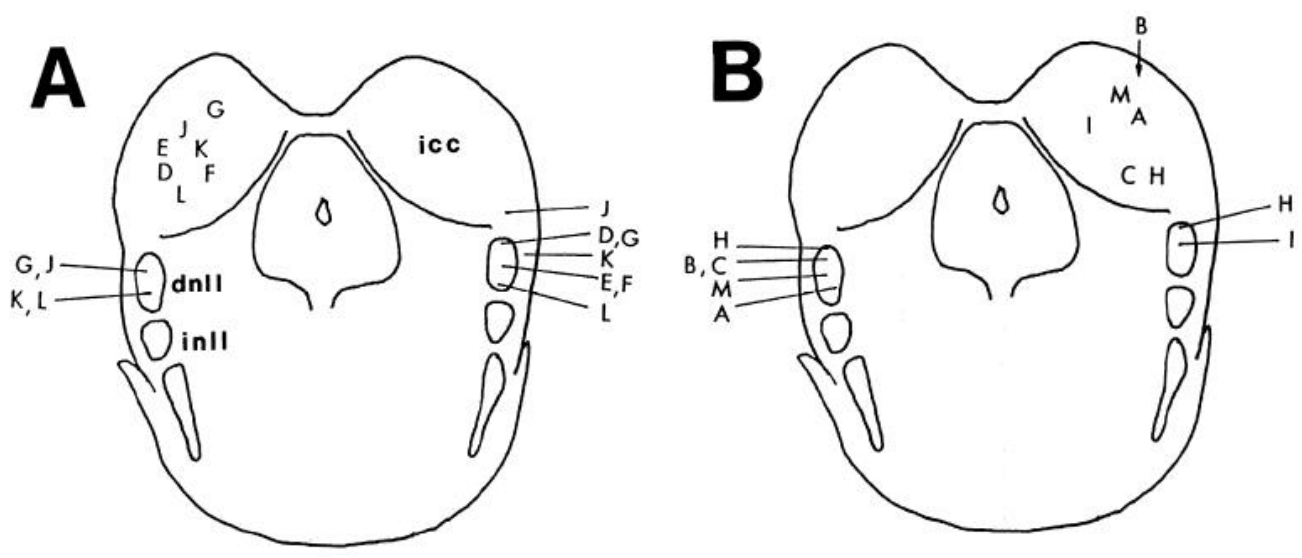

Figure 2. Schematic representation of the position of recording electrodes for cells in the left $(A)$ and right $(B)$ inferior colliculus. Each neuron in inferior colliculus is identified by a capital letter $(A-M)$. For most cells, the location of the recording electrode in the central nucleus was positively identified on the basis of a pontamine sky blue injection. The location of the electrode for cell B could not be positively identified, but the electrode track indicated that this cell was also in the central nucleus of the inferior colliculus. The position of each injection pipette ipsilateral and contralateral to the recording site is indicated by a letter $(A-M)$ corresponding to the cell from which the recording was made. Auditory structures are identified by lowercase bold letters. dnll, dorsal nucleus of the inferior colliculus; icc, central nucleus of the inferior colliculus; inll, intermediate nucleus of the lateral lemniscus. 


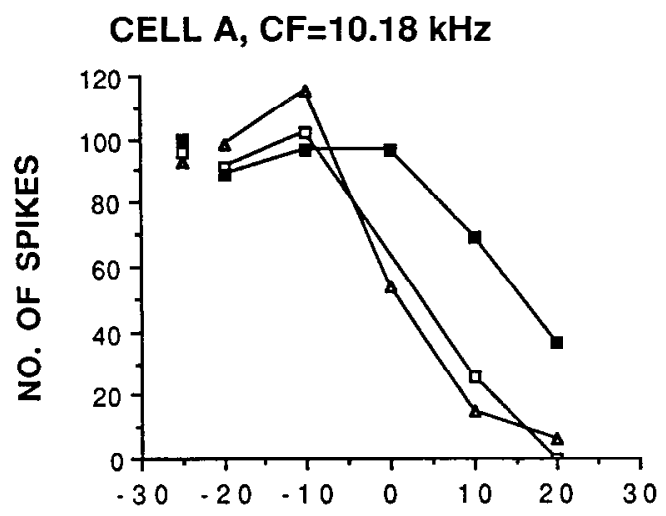

CELL $C, C F=13.08 \mathrm{kHz}$

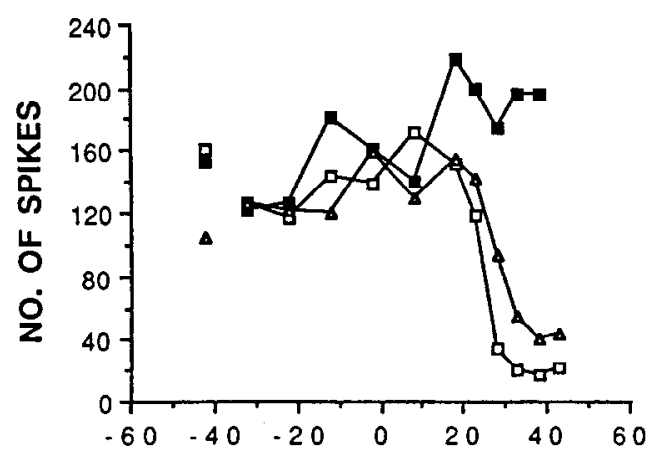

CELL E, CF=16.16 kHZ

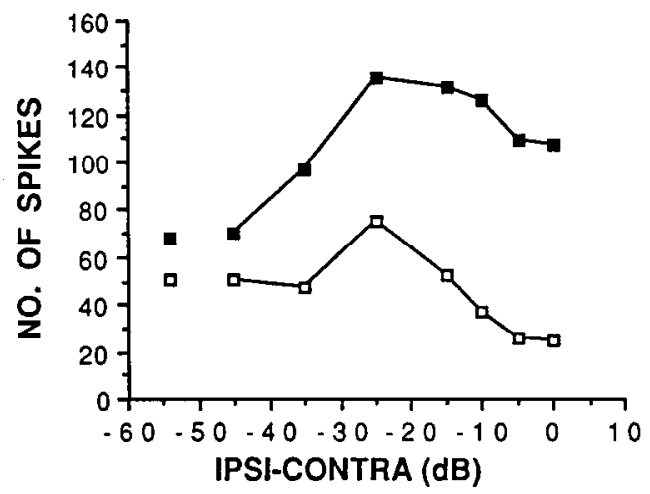

CELL B, CF $=16.33 \mathrm{kHz}$

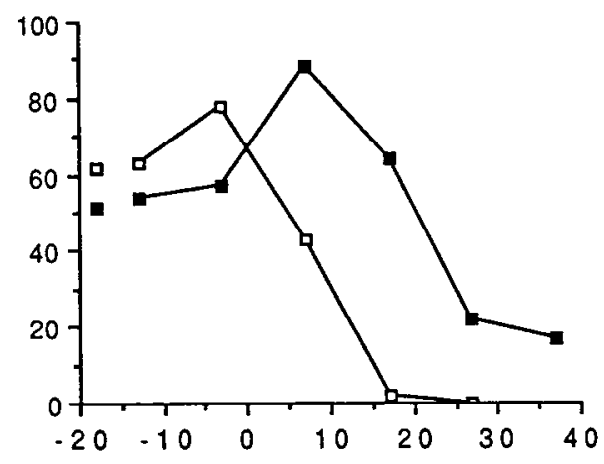

CELL $D, C F=13.52 \mathrm{kHz}$

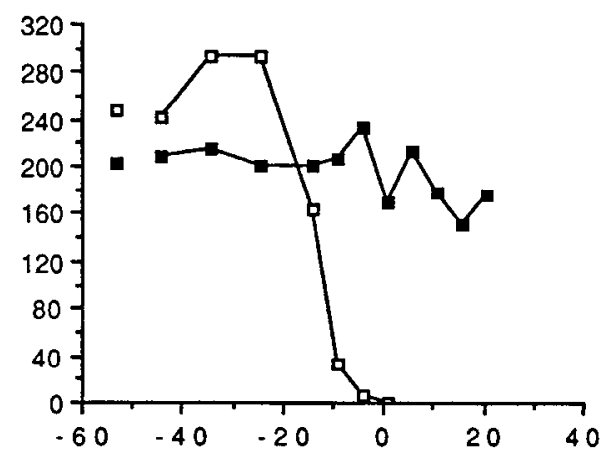

CELL F, CF $=6.05 \mathrm{kHz}$

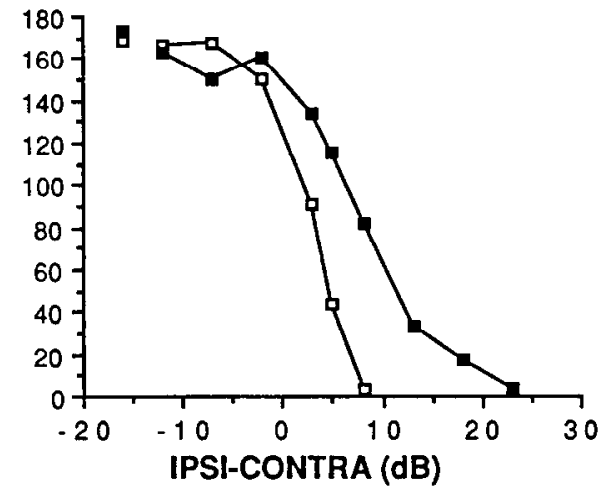

Figure 3. IID curves for six neurons in inferior colliculus before and after injection and following recovery from a kynurenic acid injection into the contralateral DNLL. Spikes are summed over 50 stimulus presentations. Symbols to the left in each panel indicate the number of spikes elicited by stimulation of the contralateral ear alone. Symbols connected by a solid line indicate the number of spikes elicited by binaural stimulation with different IIDs. Open squares, before injection; solid squares, after injection; open triangles, following recovery. letters (A-M). With the exception of cell $\mathrm{B}$, all recording sites were positively identified as located within the central nucleus of the inferior colliculus. For cell B, the pontamine sky blue was not apparent in histology, but the electrode track through the inferior colliculus was directed toward the central nucleus. Based on the success of the stereotaxic placements in other cases, it is likely that the electrode tip for this cell was also within the central nucleus. Furthermore, all neurons were narrowly tuned to sound frequency, which is characteristic of neurons in the central nucleus (Kelly et al., 1991).

The placements of injection pipettes in the DNLL are also shown in Figure 2. The exact location of the injection pipette varied slightly from one experiment to another. In some cases, the pipette tip was more dorsal and in others more ventral within the DNLL. However, most injection sites were well within the boundaries of the DNLL. For one cell (cell J), the right pipette placement was just above the DNLL within the ventral margin of the inferior colliculus. Cell $\mathbf{J}$ is included in this presentation because application of kynurenic acid at this location was found to have a distinct effect on responses in the contralateral inferior colliculus, consistent with the effects of direct injection into the DNLL.

Contralateral DNLL injections. In every neuron tested, injection of kynurenic acid into the contralateral DNLL altered the binaural response in the central nucleus of the inferior colliculus. Prior to kynurenic acid injection, all inferior colliculus neurons in our sample showed evidence of binaural suppression; that is, they were excited by contralateral stimulation and inhibited by ipsilateral stimulation. Following injection of kynurenic acid into the contralateral DNLL, the inhibitory effect was greatly reduced in every cell investigated. Six examples of this effect are shown in Figure 3. For five neurons, the response 
CELL B

Normal
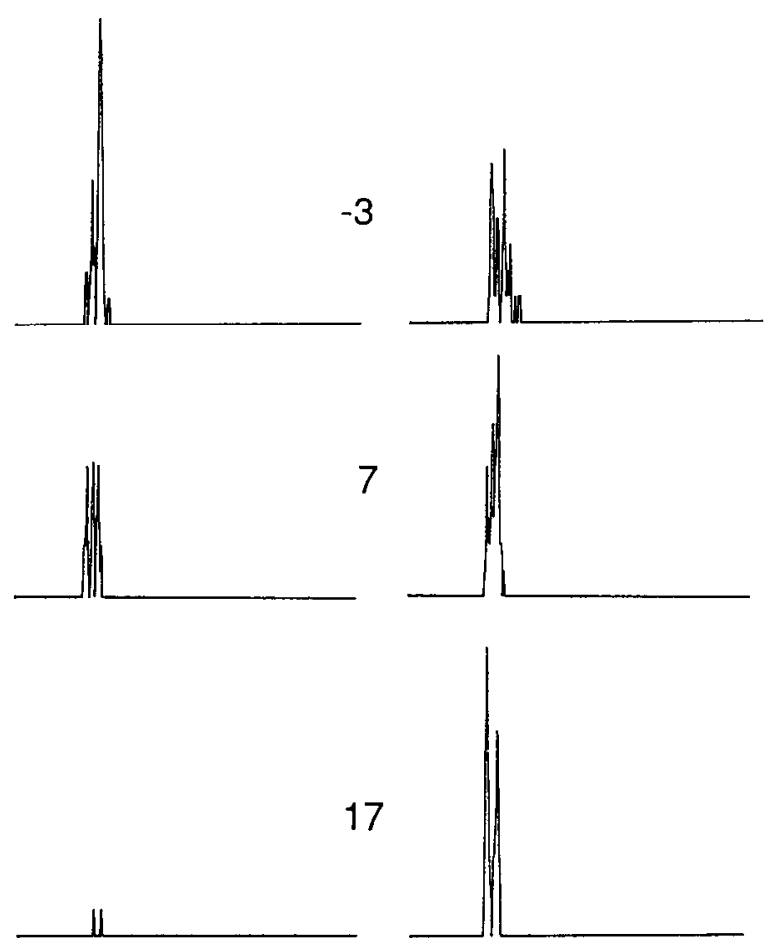

27

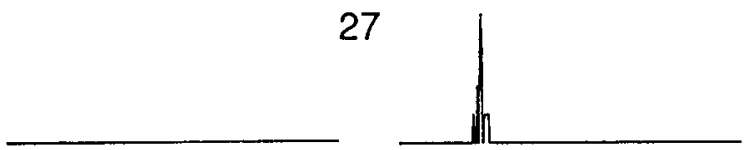

37

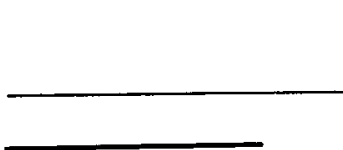

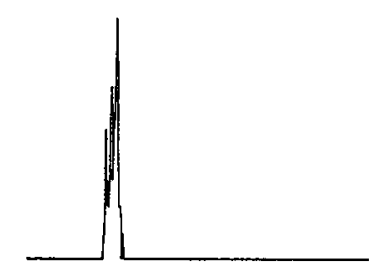

Kynurenic
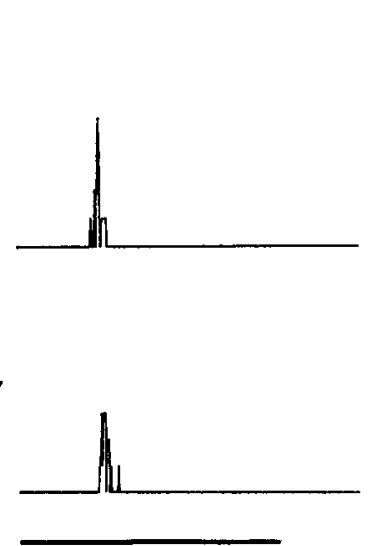

CELL D

Normal Kynurenic
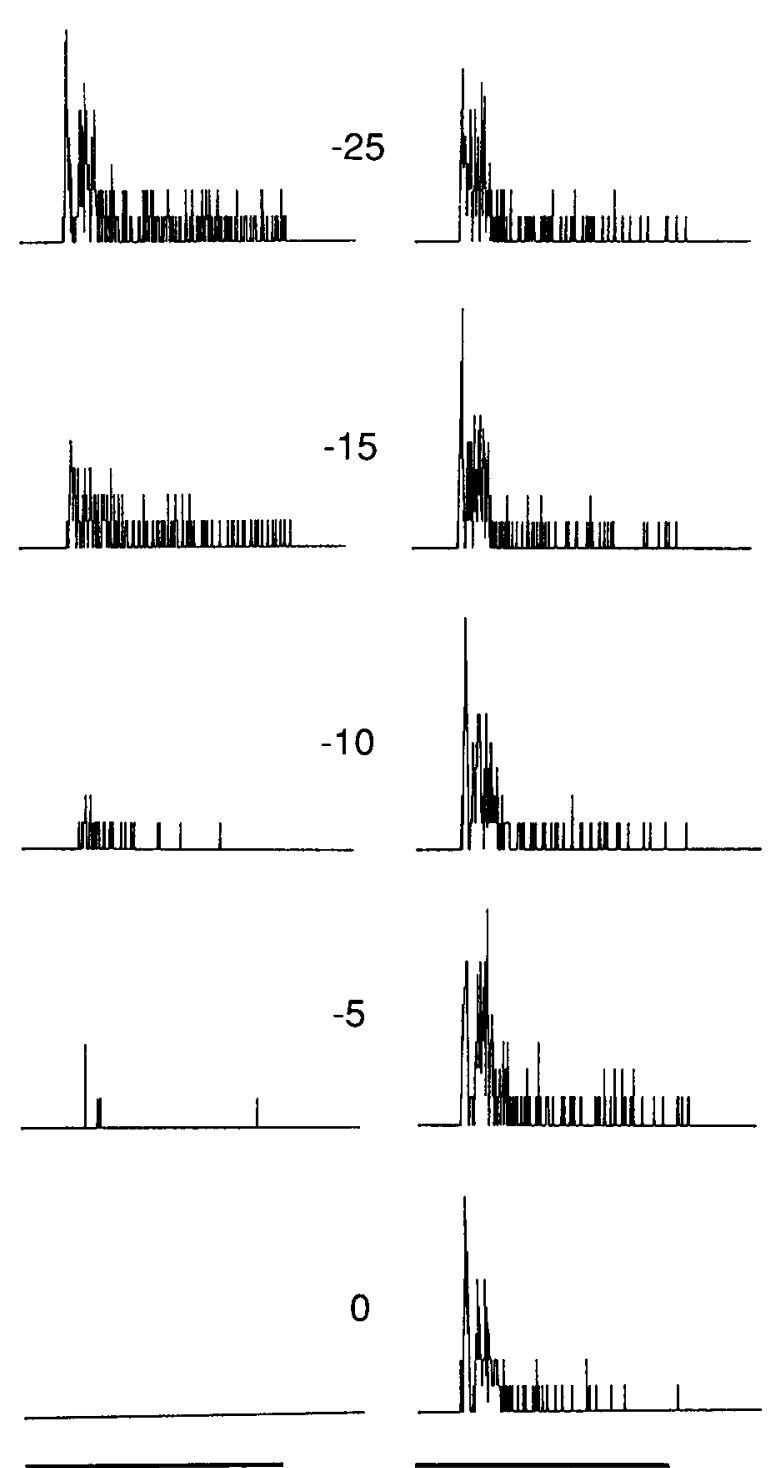

Figure 4. PSTHs for two neurons in inferior colliculus illustrating the effect of injecting kynurenic acid into the contralateral DNLL. The number of action potentials over 50 stimulus presentations is represented by the height of the graph. The response pattern is shown for a series of binaural stimuli with increasing ipsilateral sound pressure. Sound pressure is indicated beside each as the IID in dB. The magnitude of suppression can be compared under normal conditions and after injection of kynurenic acid into the contralateral DNLL for each cell. The duration of the stimulus $(110 \mathrm{msec})$ is indicated by the bar at the bottom of each column of PSTHs. For cell B, the poststimulus response pattern was onset only. For cell $\mathrm{D}$, the pattern was a typical sustained response. The kynurenic acid injection reduced the effectiveness of ipsilateral inhibition without altering the poststimulus response pattern in both cases.

pattern was dominated by binaural suppression (cells A-D and F). In each of these cases, an increase in ipsilateral sound pressure resulted in the complete, or near complete, suppression of tone-evoked activity before the kynurenic acid injection was made (open squares). Following the injection, the inhibitory effect was greatly reduced, although not completely eliminated, in three cells (A, B, and F). In two additional cells (C and D), the inhibitory effect was totally blocked, at least within the limits of our equipment for generating high-intensity sounds. Of course, it is possible that these neurons would have shown some remaining inhibition at higher ipsilateral sound pressure levels.
One neuron (cell E) exhibited an unusual binaural response pattern, at least for our sample. Prior to injection, this cell was characterized by binaural facilitation at low ipsilateral sound pressures, and relatively moderate binaural suppression at higher levels. Injection of kynurenic acid into the contralateral DNLL in this case resulted in a release from ipsilateral inhibition similar to that seen in other cells, but also unmasked a strong facilitation that was not apparent in the normal response curve.

Binaural response curves after recovery from DNLL injections were obtained in two of the cells $(\mathrm{A}$ and $\mathrm{C})$ illustrated in Figure 3. The IID curves in both cases were restored to their 

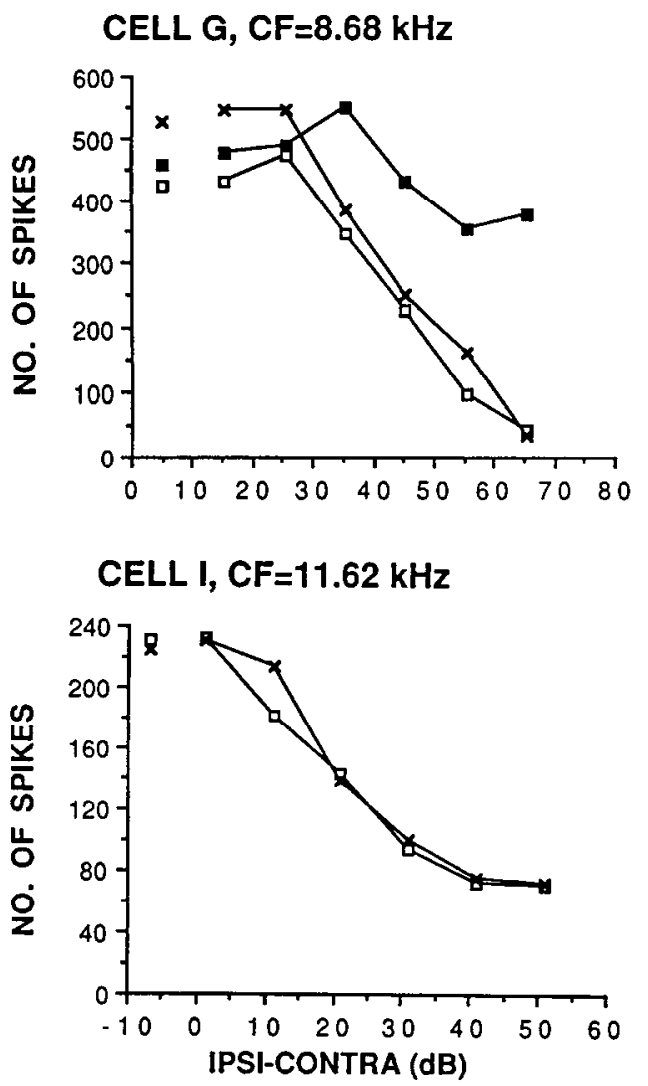

\author{
CELL H, CF $=22.43 \mathrm{kHz}$
}

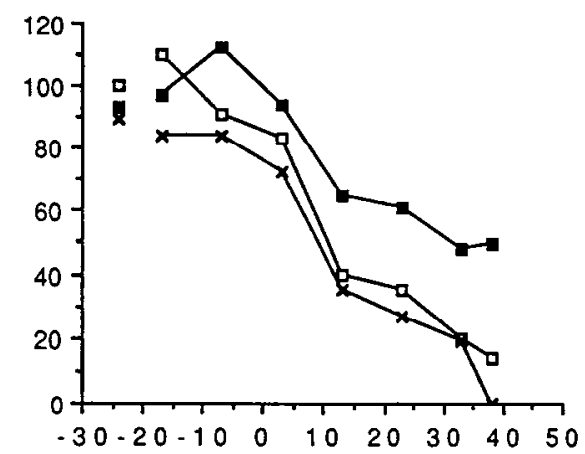

CELL J, CF $=2.45 \mathrm{kHz}$

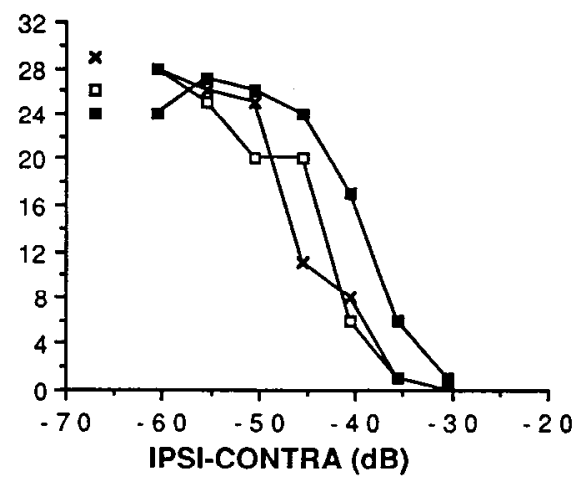

Figure 5. IID curves for neurons in the inferior colliculus illustrating the effect of kynurenic acid injection into the ipsilateral DNLL. The number of spikes is summed over 50 stimulus presentations. Symbols to the left in each panel indicate the number of spikes elicited by stimulation of the contralateral ear alone. Symbols connected by a solid line indicate the number of spikes elicted by binaural stimulation with different IIDs. Open squares, before injection; crosses. after injection into the ipsilateral DNLL; solid squares, after injection into the contralateral DNLL. normal appearance within $45 \mathrm{~min}$ after the initial kynurenic acid injection (open triangles).

The disinhibition produced by contralateral DNLL injections was not dependent on the temporal response pattern of the cell from which the recording was made. In our sample, eight neurons in the inferior colliculus normally responded to a tone burst with a transient discharge of action potentials at stimulus onset, eight responded in a sustained fashion throughout the duration of the stimulus, and four had responses with both onset and sustained components separated by a momentary pause in the production of action potentials. In no case did the contralateral DNLL injection alter the temporal pattern or selectively affect one component of the response. Both onset and sustained components were disinhibited by injection of kynurenic acid into the contralateral DNLL. Two examples of PSTHs from neurons in inferior colliculus are given in Figure 4. In one neuron (cell B), the response satisfied our definition of an onset pattern. In this case, a strong ipsilateral inhibitory effect was reduced by blocking DNLL activity, but without changing the temporal response pattern. A second neuron (cell D) serves to illustrate a typical sustained response. Again, injection of kynurenic acid into the DNLL reduced the strength of ipsilateral inhibition without altering the neuron's temporal response characteristics.

Ipsilateral DNLL injections. Injection of kynurenic acid into the ipsilateral DNLL was without effect on IID curves in every neuron tested (seven cases). The lack of effect for four representative neurons is illustrated in Figure 5. The IID function was similar before (open squares) and after (crosses) ipsilateral DNLL injection in each of these four cases. In three of the cells shown here (cells $\mathrm{G}, \mathrm{H}$, and J), the effects of contralateral injection were also tested. In each case, the contralateral injection shifted the IID function by reducing the effect of ipsilateral inhibition. It should be noted in passing that the neuron least affected by contralateral injection (cell $\mathrm{J}$ ) was one in which the injection pipette was positioned in the ventral inferior colliculus just above the DNLL. For this neuron, a slightly misplaced contralateral injection was more effective than a precisely placed ipsilateral injection.

Control conditions. The effect of injecting Locke's solution into the DNLL was investigated in several cases to determine whether the contralateral shift of binaural responses in inferior colliculus was specific to kynurenic acid. The volume and concentration of the Locke's solution were identical to those of the vehicle containing kynurenic acid. The injection of Locke's solution had no effect on the IID curves of inferior colliculus neurons. Two examples are shown in Figure 6. In each of these examples, several experimental manipulations were carried out. First, an IID curve was obtained before any injections were made (open squares). Second, an injection of kynurenic acid was made into the ipsilateral DNLL (crosses). No alteration in the IID curve was found. Third, an injection of kynurenic acid was made into the contralateral DNLL (solid squares). Under this condition, the IID curves of both cells were shifted in the direction expected from reduced ipsilateral inhibition. The outcome was similar to that already reported for contralateral DNLL injections. Fourth, the cells were allowed to recover from the effects of kynurenic acid injection (open triangles). After $40 \mathrm{~min}$, the IID curves of both cells were completely restored to their original state. Finally, Locke's solution by itself was injected into the contralateral DNLL (solid triangles). In neither case did the injection of the vehicle solution have a discernable effect on the IID curve.

Time course of recovery. In several cells, the time course of recovery was systematically investigated. In most cases, IID 
Figure 6. IID curves for neurons in the inferior colliculus illustrating various control conditions. Spikes are summed over 50 stimulus presentations. Symbols to the left in each panel indicate the number of spikes elicited by stimulation of the contralateral ear alone. Symbols connected by a solid line indicate the number of spikes elicited by binaural stimulation with different IIDs. Open squares, before kynurenic acid injection; crosses, after injection of kynurenic acid into the ipsilateral DNLL; solid squares, after injection of kynurenic acid into the contralateral DNLL; open triangles, recovery following contralateral injection; solid triangles, following injection of Locke's solution into the contralateral DNLL.
CELL K, CF $=11.98 \mathrm{kHz}$

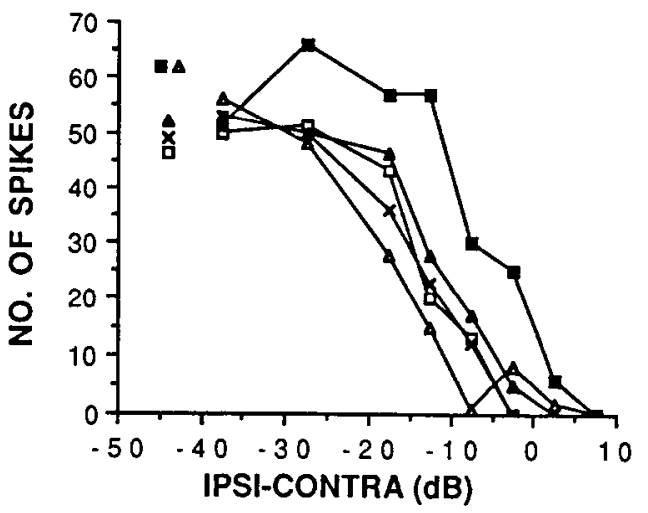

CELL L, CF $=12.62 \mathrm{kHz}$

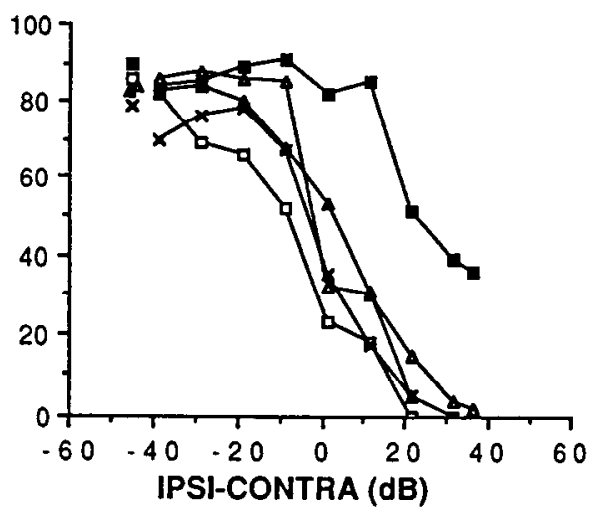

curves were obtained at various time intervals after injection and an estimate was made of the period necessary for full recovery. For most cells tested, no recovery was observed after $15 \mathrm{~min}$, partial recovery was found after $30 \mathrm{~min}$, and full recovery was evident after $45 \mathrm{~min}$ to $1 \mathrm{hr}$.

In one neuron, the course of recovery was documented in more detail. In this example (shown in Fig. 7), the ipsilateral sound pressure was set at a fixed value selected to produce strong suppression. A suppression score, based on comparison of contralateral $(C)$ and bilateral $(B)$ firing rate, was determined at various times after injection. The depth of suppression, as percentage, was defined as $[(C-B) / C] \times 100$. Before injection of kynurenic acid into the contralateral DNLL, the suppression score was around $80 \%$. Immediately after injection, the depth of suppression was reduced to less than $10 \%$. Suppression remained between 0 and $10 \%$ for $30 \mathrm{~min}$ and then began to increase gradually. After $45 \mathrm{~min}$ recovery was nearly complete.

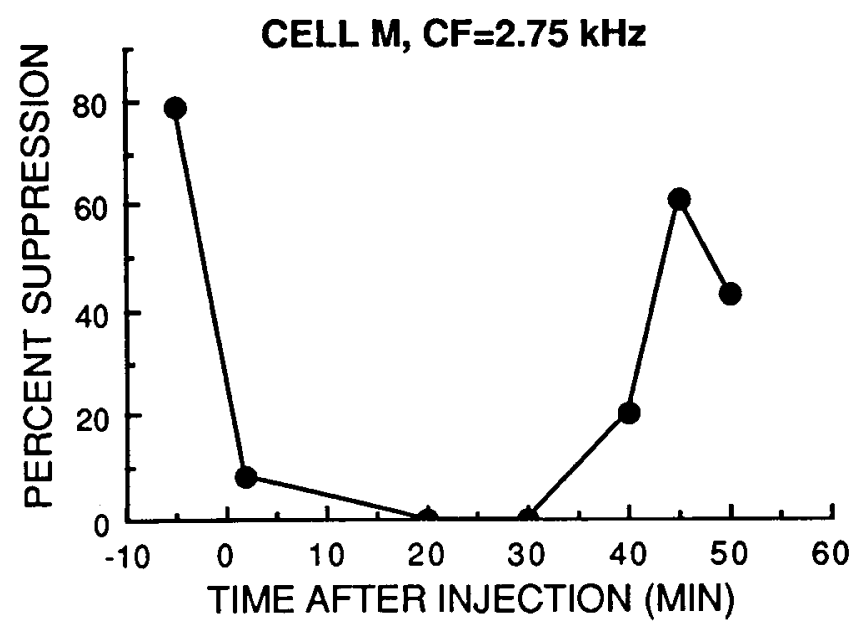

Figure 7. Time course of reversible block of binaural suppression produced by injection of kynurenic acid into the contralateral DNLL. The percentage suppression of a neuron in inferior colliculus was determined from the number of action potentials evoked by contralateral stimulation $(C)$ relative to the number evoked by bilateral stimulation $(B)$; percentage suppression was defined as $[(C-B) / C] \times 100$. The suppression drops from around $80 \%$ prior to injection to almost $0 \%$ immediately after injection. Binaural suppression for this cell gradually recovered over a period of $40 \mathrm{~min}$ to $1 \mathrm{hr}$.

\section{Discussion}

Anatomical studies. The reduction in binaural suppression in inferior colliculus following injection of kynurenic acid into the DNLL is probably due to the disruption of an inhibitory pathway from the DNLL to the contralateral DNLL and/or inferior colliculus. Immunocytochemical studies have shown that the vast majority of neurons in the DNLL are GABAergic (Adams and Mugnaini, 1984; Thompson et al., 1985; Moore and Moore, 1987; Roberts and Ribak, 1987; Glendenning and Baker, 1988). These neurons give rise to a prominent projection to the contralateral DNLL and inferior colliculus through the commissure of Probst, thus providing an anatomical basis for contralateral inhibition in the auditory system (Goldberg and Moore, 1967; Beyerl, 1978; Adams, 1979; Brunso-Bechtold et al., 1981; Glendenning et al., 1981; Kudo, 1981; Zook and Casseday, 1982, 1987; Coleman and Clerici, 1987; Shneiderman et al., 1988; Hutson et al., 1991). Electron microscopy provides further evidence that the DNLL is a source of contralateral inhibition. The terminals of DNLL neurons projecting to contralateral DNLL and inferior colliculus have flattened or pleomorphic synaptic vesicles and symmetrical synaptic contacts that are usually associated with inhibitory synapses (Oliver and Shneiderman, 1988; Shneiderman and Oliver, 1989). Thus, injection of kynurenic acid into the DNLL would have the effect of blocking inhibitory input to the contralateral inferior colliculus.

Anatomical data indicate that the contralateral inhibitory projections from DNLL may be involved in binaural processing. The terminal fields of axons from DNI.I, form bands along isofrequency contours in the contralateral inferior colliculus and interdigitate with projections from other sources including the lateral superior olive, medial superior olive, and contralateral cochlear nucleus (Henkel and Spangler, 1983; Oliver, 1984, 1987; Shneiderman and Henkel, 1987; Shneiderman et al., 1988). The convergence of afferents from multiple sources suggests the possibility that binaural interactions occur at the level of the inferior colliculus and that the DNLL plays a role in shaping binaural responses particularly of the EI type. The suggestion has been made, on the basis of anatomical data, that the DNLL plays a role in establishing the laterality of responses in the auditory pathway by exerting an inhibitory influence on the DNLL and/ or the inferior colliculus on the opposite side of the brain (Shneiderman et al., 1988). Evidence of a reversiblc block of binaural suppression in inferior colliculus by local injection of kynurenic 
acid into the contralateral DNLL is consistent with this idea.

Physiological studies. The results of the present study are also reinforced by previous physiological findings. One line of supporting evidence is that GABAergic blockers alter binaural responses in the inferior colliculus probably through connections with the contralateral DNLL. A second is that kainic acid lesions of the DNLL alter binaural responses recorded from the contralateral auditory cortex in the rat. Together, these studies show that the DNLL is important for shaping binaural responses in the central auditory system, as indicated below.

Several recent studies have demonstrated that binaural responses of cells in the inferior colliculus are altered by iontophoretic injection of the GABA $A_{A}$ antagonist bicuculline (Faingold et al., 1989, 1991; Park and Pollak, 1992). Bicuculline injection reduces the strength of inhibition produced by acoustic stimulation of the ipsilateral ear and shifts the IID curves of neurons in the inferior colliculus of both rat and mustache bat. One possible source of GABAergic innervation that might account for this effect is the contralateral DNLL. Local injection of bicuculline would likely block GABAergic terminals associated with the contralateral projection from DNLL. The shift in binaural response curves produced by iontophoretic injection of bicuculline is similar to that produced by a reversible block of excitatory activity in the contralateral DNLL with kynurenic acid.

Iontophoretic injection of bicuculline also produces effects on monaural responses in inferior colliculus that may be independent of the contralateral GABAergic projection from DNLL (Faingold et al., 1989; Pollak and Park, 1992). For example, in the bat, the local application of bicuculline can alter monaural rate-intensity functions, converting a nonmonotonic into a monotonic response. Also, bicuculline can result in the broadening of frequency response tuning curves of monaurally driven cells. It is likely that these effects are due, at least in part, to GABAergic projections from sources other than the contralateral DNLL. Physiological responses of neurons in the inferior colliculus may be affected by GABAergic innervation from ipsilateral as well as contralateral DNLL, or by intrinsic GABAergic interneurons forming local circuits within the inferior colliculus. In contrast, the effects of kynurenic acid injection into the DNLL are probably due solely to disruption of the contralateral GABAergic projection.

Unilateral kainic acid lesions of DNLL produce a permanent change in binaural response functions recorded from contralateral auditory cortex (Glenn and Kelly, 1991, 1992). The amplitude of the auditory cortical evoked response in the rat is highly dependent on binaural timing and reflects binaural interactions occurring at lower levels of the auditory pathway. Normally, the cortical response amplitude is greatest for binaural clicks in which the leading stimulus is presented to the contralateral ear. As the ITD is gradually shifted in favor of the ipsilateral ear, the response amplitude is progressively reduced over a dynamic range of about $500 \mu \mathrm{sec}$. Following a unilateral kainic acid lesion of DNLL, the slope of the ITD curve in the hemisphere contralateral to the lesion is greatly reduced. There is no change in the maximum response amplitude, but rather, the suppression of response amplitude normally associated with ipsilateral stimulation is diminished. The most probable cause of this effect is the disruption of inhibitory projections from GABAergic neurons in DNLL to contralateral DNLL and/or inferior colliculus. The results of the present study support the evoked potential data by showing that a temporary block of neural activity in DNLL results in an alteration of binaural response curves in the central nucleus of the inferior colliculus, and that the changes are associated with a reduction of inhibition caused by stimulation of the ipsilateral ear.

Injection of kynurenic acid into the ipsilateral DNLL had no obvious effect on IID curves in the inferior colliculus. This result is significant in light of the fact that the DNLL does have a substantial anatomical projection to the ipsilateral inferior colliculus. The ipsilateral projection differs from the contralateral in several respects that might account for its apparent lack of involvement in binaural processing. First, many of the terminals of DNLL neurons that project to the ipsilateral inferior colliculus contain round synaptic vesicles, usually associated with excitatory function, whereas the overwhelming majority, if not all, of the terminals projecting to the contralateral inferior colliculus contain pleomorphic vesicles (Shneiderman and Oliver, 1989). This result suggests that the ipsilateral and contralateral projections from DNLL involve different synaptic mechanisms. Second, electrically evoked release of GABA from the guinea pig inferior colliculus, studied in vitro, is more profoundly affected by contralateral than ipsilateral DNLL lesions (Potashner et al., 1991). In animals with a previously placed unilateral kainic acid lesion of DNLL, evoked GABA levels were reduced by $50 \%$ in the contralatcral inferior colliculus, compared to only $25 \%$ in the ipsilateral inferior colliculus. These data suggest that there is a functional difference in the strength of inhibition produced by ipsilateral and contralateral pathways from DNLL to inferior colliculus. Third, the ipsilateral and contralateral projections to the inferior colliculus arise from separate populations of neurons in the DNLL. Only a very small percentage of the neurons in DNLL have collateral projections to both ipsilateral and contralateral inferior colliculus (Hutson et al., 1991). Therefore, it is possible that ipsilateral and contralateral projections are activated under different circumstances and are involved in quite different physiological functions.

The lack of ipsilateral effect is consistent with our previous investigation of kainic acid lesions in DNLL. In that study, the curves describing evoked response amplitude in auditory cortex as a function of ITD were completely unaffected in the hemisphere ipsilateral to the lesion, cven though the slopes of the ITD curves were reduced in the hemisphere contralateral to the lesion. Thus, neither cortical evoked potentials nor single-unit recordings from inferior colliculus show any effect of DNLL disruption on binaural processing in the ipsilateral auditory pathway.

Parallel pathways in binaural processing. The results of the present study suggest that parallel pathways contribute to binaural responses in the inferior colliculus. The contribution of the DNLL itself is apparent from the fact that IID curves in the inferior colliculus are shifted and the depth of ipsilateral inhibition is reduced following contralateral injection of kynurenic acid. The contribution of other pathways, independent of DNLL, is evidenced by the fact that a contralateral kynurenic acid injection does not completely eliminate binaural responses in inferior colliculus. In 17 out of the 19 cells tested in the present study, ipsilateral acoustic stimulation was still capable of producing binaural suppression in spite of contralateral DNLL injections. For two neurons that showed no binaural suppression after DNLL injection (cells C and D), some evidence of inhibition might have emerged at higher ipsilateral sound levels. The presence of some binaural response after contralateral kynurenic acid injections is consistent with the previously reported 
effects of kainic acid lesions of DNLL (Glenn and Kelly, 1991, 1992). Kainic acid lesions alter, but do not completely eliminate, binaural responses in the contralateral primary auditory cortex. Thus, neither temporary nor permanent disruption of DNLL is sufficient to exclude all binaural suppression responses. These results imply that the DNLL is not the only structure contributing to binaural suppression in the inferior colliculus. Binaural responses are probably shaped in part by additional pathways to the central nucleus of the inferior colliculus.

One possible source of hinaural interaction independent of the DNLL is the superior olivary complex. It is well known that EI binaural interactions occur in both lateral and medial superior olive (Boudreau and Tsuchitani, 1968; Goldberg and Brown, 1969; Tsuchitani and Boudreau, 1969; Guinan et al., 1972a,b; Harnischfeger et al., 1975; Inbody and Feng, 1981; Caird and Klinke, 1983; Yin and Chan, 1990). The cells in the lateral superior olive are typically excited by ipsilateral stimulation and inhibited by contralateral stimulation (Boudreau and Tsuchitani, 1968; Tsuchitani and Boudreau, 1969; Caird and Klinke, 1983). Since these cells give rise to a prominent excitatory projection to the contralateral inferior colliculus, they almost certainly make some contribution to binaural suppression responses in that nucleus, but with a reversal in the laterality of excitatory and inhibitory cffects (Glendenning and Mastcrton, 1983; Glendenning et al., 1985). Binaural suppression is also present in the medial superior olive, typically, with contralateral stimulation producing excitation and ipsilateral stimulation producing inhibition (Goldberg and Brown, 1969; Harnischfeger et al., 1975; Inbody and Feng, 1981; Yin and Chan, 1990). Since the medial superior olive has an almost exclusively ipsilateral projection to the inferior colliculus in the rat and other mammals, this pattern might also contribute to binaural suppression responses independent of DNLL (Beyerl, 1978; Adams, 1979; Coleman and Clerici, 1987). In addition, many of the cells in the lateral superior olive are glycinergic and send a substantial ipsilateral projection to the inferior colliculus (Hutson et al., 1987; Saint Marie et al., 1989; Saint Marie and Baker, 1990). This pathway would provide a possible avenue for ipsilateral inhibition of activity in the inferior colliculus. Functionally, the ipsilateral glycincrgic projection from the lateral superior olive and the contralateral GABAergic projection from DNLL are convergent and would be expected to have similar physiological effects on cells in the inferior colliculus. For example, a lateralized sound source would preferentially excite the ipsilateral lateral superior olive, which would in turn exert an inhibitory effect on the ipsilateral inferior colliculus. The same sound source would activate the contralateral DNLL and produce ipsilateral inhibition in the inferior colliculus via the crossed connections of the commissure of Probst. Thus, the ipsilateral glycinergic projection from lateral superior olive and the contralateral GABAergic projection from DNLL would make similar contributions to binaural suppression responses in inferior colliculus.

There are two possible ways in which the DNLL might modulate binaural responses in the inferior colliculus: either through its connections with the superior olivary complex, or through connections that bypass the superior olive. Recent studies have shown that binaural responses in the inferior colliculus persist following kainic acid lesions of the superior olivary complex (Kelly and Li, 1992; Sally and Kelly, 1992; Li and Kelly, 1992). This result has led to the suggestion that the remaining binaural interactions are mediated through a pathway from the contralateral cochlear nucleus to DNLL independent of the superior olive ( $\mathrm{Li}$ and Kelly, 1992). On the other hand, the DNLL receives a substantial projection from both medial and lateral superior olive, each of which provides the basis for binaural processing early in the auditory pathway. Electrophysiological studies indicate that the large majority of neurons in DNLL are sensitive to binaural input and are preferentially driven by binaural differences favoring the contralateral ear (Brugge, 1970). The binaural activity of these neurons probably reflects to a large extent the afferent in put from the superior olivary complex. Whether excitation in DNI I, originates from connections with the superior olive or arises from projections that bypass the olivary complex, the GABAergic pathway from DNLL to the opposite side of the brain makes a unique contribution to binaural processing. Increased activity in DNLL results in the suppression of responses in the contralateral inferior colliculus and, in that way, maintains or reinforces the functional laterality of the auditory pathway.

In summary, parallel pathways may be involved in controlling binaural responses in the inferior colliculus. The superior olivary complex may make a contribution independent of the DNLL. The DNLL may make its own contribution independent of the superior olive. Finally, the DNLL may make a contribution through its connections with the nuclei of the superior olivary complex. The DNLL plays an important role in shaping the binaural response characteristics of neurons located in the central nucleus of the inferior colliculus.

\section{References}

Adams JC (1979) Ascending projections to the inferior colliculus. J Comp Neurol 183:519-538.

Adams JC, Mugnaini E (1984) Dorsal nucleus of the lateral lemniscus: a nucleus of GABAergic projection neurons. Brain Res Bull 13:585590.

Aitkin LM, Anderson DJ, Brugge JF (1970) Tonotopic organization and discharge characteristics of single neurons in nuclei of the lateral lemniscus of the cat. J Neurophysiol 33:421-440.

Beyerl BD (1978) Afferent projections to the central nucleus of the inferior colliculus in the rat. Brain Res 145:209-223.

Boudreau JC, Tsuchitani C (1968) Binaural interaction in the cat superior olive S segment. J Neurophysiol 31:442-454.

Brugge JF, Anderson DJ, Aitkin LM (1970) Responses of neurons in the dorsal nucleus of the lateral lemniscus of the cat to binaural tonal stimulation. J Neurophysiol 33:441-458.

Brunso-Bechtold JK, Thompson GC, Masterton RB (1981) HRP study of the organization of auditory afferents ascending to the central nucleus of the inferior colliculus in cat. J Comp Neurol 197:705-722.

Caird D, Klinke R (1983) Processing of binaural stimuli by cat superior olivary complex neurons. Exp Brain Res 52:385-399.

Coleman JR, Clerici WJ (1987) Source of projections to subdivisions of the inferior colliculus in the rat. J Comp Neurol 262:215-226.

Faingold CL, Gehlbach G, Caspary DM (1989) On the role of GABA as an inhibitory neurotransmitter in inferior colliculus neurons: iontophoretic studies. Brain Res 500:302-312.

Faingold CL, Boersma Anderson CA, Caspary DM (1991) Involvement of GABA in acoustically-evoked inhibition in inferior colliculus neurons. Hearing Res 52:201-216.

Glendenning KK, Baker BN (1988) Neuroanatomical distribution of receptors for three potential inhibitory neurotransmitters in the brainstem auditory nuclei of the cat. J Comp Neurol 275:288-308.

Glendenning KK, Masterton RB (1983) Acoustic chiasm: efferent projections of the lateral superior olive. J Neurosci 3:1521-1537.

Glendenning KK, Brunso-Bechtold JK, Thompson GC, Masterton RB (1981) Ascending auditory afferents to the nuclei of the lateral lemniscus. J Comp Neurol 197:673-703.

Glendenning KK, Hutson KA, Nudo RJ, Masterton RB (1985) Acoustic chiasm. II: Anatomical basis of binaurality in lateral superior olive of cat. J Comp Neurol 232:261-285.

Glenn SL, Kelly JB (1991) Effects of kainic acid lesions of dorsal 
nucleus of lateral lemniscus on cortical auditory evoked responses: interaural time delays. Assoc Res Otolaryngol Abstr 14:22.

Glenn SL. Kelly JB (1992) Kainic acid lesions of the dorsal nucleus of the lateral lemniscus: effects on binaural evoked responses in rat auditory cortex. J Neurosci 12:3688-3699.

Goldberg JM. Brown PB (1969) Response of binaural neurons of dog superior olivary complex to dichotic tonal stimuli: some physiological mechanisms of sound localization. J Neurophysiol 32:613-636.

Goldberg JM, Moore RY (1967) Ascending projections of the lateral lemniscus in the cat and monkey. J Comp Neurol 129:143-156.

Guinan JJ Jr, Guinan SS. Norris BE (1972a) Single auditory units in the superior olivary complex. I. Responses to sounds and classifications based on physiological properties. Int J Neurosci 4:101-120.

Guinan JJ Jr, Norris BE, Guinan SS (1972b) Single auditory units in the superior olivary complex. II. Locations of unit categories and tonotopic organization. Int J Neurosci 4:147-166.

Hamischfeger G. Neuweiler G. Schlegel P (1975) Interaural time and intensity coding in superior olivary complex and inferior colliculus of the echolocating bat Molossus ater. J Neurophysiol 53:89-109.

Henkel CK, Spangler KM (1983) Organization of the efferent projections of the medial superior olivary nucleus in the cat as revealed by HRP and autoradiographic tracing methods. J Comp Neurol 221: $416-428$.

Hutson KA, Glendenning KK, Masterton RB (1987) Biochemical basis for the acoustic chiasm? Soc Neurosci Abstr 13:548.

Hutson KA, Glendenning KK, Masterton RB (1991) Acoustic chiasm. IV: Eight midbrain decussations of the auditory system. J Comp Neurol 312:105-131.

Inbody SB, Feng AS (1981) Binaural response characteristics of single neurons in the medial superior olivary nucleus of the albino rat. Brain Res 210:36!-366.

Kelly JB, Li L (1992) Response propertics of ncurons in the rat's inferior colliculus following kainic acid lesions of the superior olivary complex: interaural intensity difference functions. Assoc Res Otolaryngol Abstr 15:61.

Kelly JB, Glenn SL, Beaver CJ (1991) Sound frequency and binaural response properties of single neurons in rat inferior colliculus. Hearing Res 56:273-280.

Kudo $M$ (1981) Projections of the nuclei of the lateral lemniscus in the cat: an autoradiographic study. Brain Res 221:57-69.

Li L. Kelly JB (1992) Binaural responses in rat inferior colliculus following kainic acid lesions of the superior olive: interaural intensity difference functions. Hearing Res 61:73-85.

Moore JK. Moore RY (1987) Glutamic acid decarboxylase-like immunoreactivity in brainstem auditory nuclei of the rat. J Comp Neurol 260:157-174.

Nelson PG, Erulkar SD (1966) Synaptic mechanisms of excitation and inhibition in the central auditory pathway. J Neurophysiol 26:908923.

Oliver DL (1984) Dorsal cochlear nucleus projections to the inferior colliculus in the cat: a light and electron microscopic study. J Comp Neurol 224:155-172.
Oliver DL (1987) Projections to the inferior colliculus from the anteroventral cochlear nucleus in the cat: possible substrates for binaural interaction. J Comp Neurol 264:24-46.

Oliver DL, Shneiderman A (1989) An EM study of the dorsal nucleus of the lateral lemniscus: inhibitory, commissural. synaptic connections between ascending auditory pathways. J Neurosci 9:967-982.

Park TJ. Pollak GD (1992) The role of GABA in shaping binaural response properties and receptive fields of E-I neurons in the inferior colliculus. Assoc Res Otolaryngol Abstr 15:79.

Pollak GD, Park TJ (1992) The role of GABA in shaping monaural properties of neurons in the inferior colliculus. Assoc Res Otolaryngol Abstr 15:79.

Potashner SJ, Shneiderman A, Chase MB, Benson C, Rockwood JM (1991) GABA and glycine release from guinea pig inferior colliculus after ablation of dorsal nucleus of lateral lemniscus. Soc Neurosci Abstr 17:300.

Roberts RC, Ribak CE (1987) GABAergic neurons and axon terminals in the brainstem auditory nuclei of the gerbil. J Comp Neurol 258: 267-280.

Saint Marie RL, Baker RA (1990) Neurotransmitter-specific uptake of ['H]glycine from the inferior colliculus by ipsilateral projections of the superior olivary complex and nuclei of the lateral lemniscus. Brain Res 524:244-253.

Saint Marie RL, Ostapoff EM, Morest DK, Wenthold RJ (1989) Glycine-immunoreactive projection of the cat lateral superior olive: possible role in midbrain ear dominance. $J$ Comp Neurol 279:382-396.

Sally SL, Kelly JB (1992) Effects of superior olivary complex lesions on binaural responses in rat inferior colliculus. Brain Res 572:5-18.

Shneiderman A, Henkel CK (1987) Banding of lateral superior olivary nucleus afferents in the inferior colliculus: a possible substrate for sensory integration. J Comp Neurol 266:519-534.

Shneiderman A, Oliver DL (1989) EM autoradiographic study of the projections from the dorsal nucleus of the lateral lemniscus: a possible source of inhibitory inputs to the inferior colliculus. J Comp Neurol 286:28-47.

Shneiderman A, Oliver DL, Henkel CK (1988) Connections of the dorsal nucleus of the lateral lemniscus: an inhibitory parallel pathway in the ascending auditory system? J Comp Neurol 276:188-208.

Thompson GC, Cortez AM. Lam DM (1985) Localization of GABA immunoreactivity in the auditory brainstem of guinea pigs. Brain Res 339:119-122.

Tsuchitani C, Boudreau JC (1969) Stimulus level of dichotically presented tones and cat superior olive $\mathbf{S}$-segment cell discharge. J Acoust Soc Am 46:978-988.

Yin TCT, Chan JCK (1990) Interaural time sensitivity in medial superior olive of cat. J Neurophysiol 64:465-488.

Zook JM. Casseday JH (1982) Origin of ascending projections to the inferior colliculus in the mustache bat, Pteronotus parnelli. J Comp Neurol 207:14-28.

Zook JM, Casseday JH (1987) Convergence of ascending pathways at the inferior colliculus of the mustache bat. Pteronotus parnelli. J Comp Neurol 261:347-361. 